\title{
$M$-Constants, Dominguez-Benavides
} coefficient, and weak fixed point property in Orlicz sequence spaces equipped with the $p$-Amemiya norm

\author{
Yunan Cui ${ }^{1}$, Henryk Hudzik ${ }^{2}$ and Marek Wisła ${ }^{2 *}$
}

\author{
"Correspondence: \\ marek.wisla@amu.edu.pl \\ ${ }^{2}$ Faculty of Mathematics and \\ Computer Science, Adam \\ Mickiewicz University in Poznań, \\ ul. Umultowska 87, Poznań, 61-614, \\ Poland \\ Full list of author information is \\ available at the end of the article
}

\begin{abstract}
In this paper, a formula for the $M$-constants $\mu_{n}\left(/_{\Phi, p}\right)$ of Orlicz sequence spaces $/_{\Phi, p}$ equipped with the $p$-Amemiya norm as well as for the equalities $\mu_{n}\left(l_{\Phi, p}\right)=n$ for any $n \in \mathcal{N}$ are given. Moreover, the Fatou property, weak orthogonality, and the weak fixed point property of these spaces are discussed. Finally a direct formula for the Dominguez-Benavides coefficient of the spaces $/_{\Phi, p}$ is given in terms of the Orlicz function generating these spaces. Both, $M$-constants and Dominguez-Benavides coefficient, are strongly related to the fixed point theory.

MSC: 46E30; 46B20; 47H10

Keywords: $M$-constants; normal structure; weakly normal structure; Orlicz sequence space; $p$-Amemiya norm; almost isometric copy of $\ell^{1}$; fixed point property; weak fixed point property; weak orthogonality; Garcia-Falset coefficient; Dominguez-Benavides coefficient
\end{abstract}

\section{Introduction and preliminaries}

In the following, $X$ and $X^{*}$ will stand for a Banach space and its dual space, respectively. By $S(X)$ and $B(X)$ we will denote the unit sphere and the unit ball of $X$, respectively. For a nonempty subset $C$ of $X$, a mapping $T: C \rightarrow C$ is said to be nonexpansive provided the inequality

$$
\|T x-T y\| \leq\|x-y\|
$$

holds for every $x, y \in C$. A Banach space $X$ is said to have the fixed point property (resp., weak fixed point property) if for every nonempty bounded closed (resp. weakly compact) convex subset of $C \subset X$ the nonexpansive mapping $T: C \rightarrow C$ has a fixed point in $C$ (see, e.g., $[1,2])$.

Many properties of Banach lattices related to their isomorphic or isometric structure, depend on a behavior of some numerical characteristics called indices (Boyd indices, Gröbler indices, type, cotype etc.). In the theory of Banach lattices the so called 
$M$-constants defined by

$$
\mu_{n}(X)=\sup \left\{\left\|\bigvee_{i=1}^{n} x_{i}\right\|: 0 \leq x_{i} \in X,\left\|x_{i}\right\| \leq 1 \text { for } i=1,2, \ldots, n\right\}
$$

are also used (see, e.g., [3-5]). The numbers $\mu_{n}(X)$ are useful in the lattice isomorphic classification of special types of Banach lattices. It is well known that, in the definition of $\mu_{n}(X)$, we can restrict ourselves to pairwise orthogonal elements (see [6]).

The constant $\mu_{2}(X)$, called the Riesz angle, plays an important role in the fixed point theory. It is well known that weakly orthogonal Banach lattices (for the definition of this property see Section 3) with $\mu_{2}(X)<2$ have the weak fixed point property (see [7]).

Let $\Phi: \mathcal{R} \rightarrow[0, \infty)$ be an Orlicz function, i.e., let $\Phi$ be an even, convex function with $\Phi(0)=0$ and $\lim _{u \rightarrow \infty} \Phi(u)=\infty$. We say that an Orlicz function $\Phi$ satisfies the $\delta_{2}$-condition (shortly: $\Phi \in \delta_{2}$ ), if there are positive constants $u_{0}$ with $0<\Phi\left(u_{0}\right)<\infty$ and $K>0$ such that $\Phi(2 u) \leq K \Phi(u)$ for all $|u| \leq u_{0}$. Note that $\Phi \in \delta_{2}$ implies immediately that $\Phi(u)>0$ for every $u \neq 0$.

By $\Psi$ we will denote the function complementary to $\Phi$ in the sense of Young, i.e., the function $\Psi$ is defined by the formula

$$
\Psi(u)=\sup \{|u| v-\Phi(v): v \geq 0\} .
$$

Let $l^{0}$ be the space of all real sequences $x=(x(i))$. For a given Orlicz function $\Phi$ we define on $l^{0}$ a convex functional (called a pseudomodular) by

$$
I_{\Phi}(x)=\sum_{i=1}^{\infty} \Phi(x(i))
$$

where $x=(x(i)) \in l^{0}$. By the Orlicz space $l_{\Phi}$ generated by the Orlicz function $\Phi$ we mean the vector space

$$
l_{\Phi}=\left\{x \in l^{0}: I_{\Phi}(c x)<\infty \text { for some } c>0 \text { depending on } x\right\} .
$$

Further, by the space $h_{\Phi}$ of finite elements (= order continuous elements) we will mean the vector space

$$
h_{\Phi}=\left\{x \in l^{0}: \forall \lambda>0 \sum_{i=i_{1}}^{\infty} \Phi\left(\lambda x_{i}\right)<\infty\right\} .
$$

These spaces are usually equipped with the Luxemburg norm (see [8-10])

$$
\|x\|_{\Phi}=\inf \left\{\lambda>0: I_{\Phi}\left(\frac{x}{\lambda}\right) \leq 1\right\}
$$

or with the equivalent one

$$
\|x\|_{\Phi}^{0}=\sup \left\{\sum_{i=1}^{\infty}|x(i) y(i)|: y \in l_{\Psi}, l_{\Psi}(y) \leq 1\right\}
$$


called the Orlicz norm (see $[11,12])$. The Orlicz norm can equivalently be expressed by the Amemiya formula (see [13])

$$
\|x\|_{\Phi}^{0}=\inf _{k>0} \frac{1}{k}\left(1+I_{\Phi}(k x)\right) .
$$

The last formula can be generalized to a family of norms (called $p$-Amemiya norms) depending on the parameter $1 \leq p \leq \infty$ in the following way (see $[13,14]$ ):

$$
\|x\|_{\Phi, p}=\inf _{k>0} \frac{1}{k} s_{p}\left(I_{\Phi}(k x)\right)
$$

where

$$
s_{p}(u)= \begin{cases}\left(1+u^{p}\right)^{\frac{1}{p}} & \text { for } 1 \leq p<\infty, \\ \max \{1, u\} & \text { for } p=\infty\end{cases}
$$

Evidently $\|x\|_{\Phi, 1}=\|x\|_{\Phi}^{0}$ and it is easy to prove that the Luxemburg norm and the $\infty$-Amemiya norm coincide as well, i.e., $\|x\|_{\Phi, \infty}=\|x\|_{\Phi}$ (see, e.g., [14]). The $p$-Amemiya norms have the following relationships (for all $1 \leq p^{\prime} \leq p \leq \infty$ ):

$$
\|x\|_{\Phi}=\|x\|_{\Phi, \infty} \leq\|x\|_{\Phi, p} \leq\|x\|_{\Phi, p^{\prime}} \leq\|x\|_{\Phi, 1}=\|x\|_{\Phi}^{0} \leq 2\|x\|_{\Phi}
$$

and

$$
\|x\|_{\Phi} \leq\|x\|_{\Phi, p} \leq 2^{1 / p}\|x\|_{\Phi} .
$$

We will write $l_{\Phi, p}$ when we would like to underline that the Orlicz space $l_{\Phi}$ is equipped with the $p$-Amemiya norm $\|\cdot\|_{\Phi, p}$.

This paper is a continuation of investigations of geometric properties of Orlicz spaces equipped with the $p$-Amemiya norm over the atomless measure space presented in [14$18]$.

\section{$2 M$-Constants and order isometric copies of $\ell_{1}$ in $I_{\Phi, p}$ spaces}

In the following, by $S_{0}\left(l_{\Phi, p}\right)$ we will mean the subset of the unit sphere $S\left(l_{\Phi, p}\right)$ that consists of sequences with finite support only.

Theorem 2.1 For any $1 \leq p \leq \infty, n \in \mathcal{N}$, and any Orlicz function $\Phi$,

$$
\mu_{n}\left(l_{\Phi, p}\right)=\sup \left\{d_{x_{1}, \ldots, x_{n}}: x_{1}, \ldots, x_{n} \in S_{0}\left(l_{\Phi, p}\right) \text { and } x_{j} \wedge x_{j^{\prime}}=0 \text { for } j \neq j^{\prime}\right\}
$$

where the numbers $d_{x_{1}, \ldots, x_{n}}>0$ are defined by

$$
d_{x_{1}, \ldots, x_{n}}=\inf _{k>1}\left\{d_{x_{1}, \ldots, x_{n}, k}: \frac{1}{k} s_{p}\left(\sum_{j=1}^{n} I_{\Phi}\left(\frac{k x_{j}}{d_{x_{1}, \ldots, x_{n}, k}}\right)\right)=1\right\} .
$$

Proof Let $x_{1}, \ldots, x_{n} \in S_{0}\left(l_{\Phi, p}\right), x_{j} \wedge x_{j^{\prime}}=0$ for $j \neq j^{\prime}$ and let $k>1$. Since the set $\left\{i \in \mathcal{N}: x_{j}(i) \neq\right.$ $0,1 \leq j \leq n\}$ is finite, the function $\Phi$ is continuous and attains the value 1 (because it takes 
only finite values), there exists $d_{x_{1}, \ldots, x_{n}, k}>0$ such that

$$
\frac{1}{k} s_{p}\left(\sum_{j=1}^{n} I_{\Phi}\left(\frac{k x_{j}}{d_{x_{1}, \ldots, x_{n}, k}}\right)\right)=1 .
$$

Note that, for every $x_{1}, \ldots, x_{n} \in S_{0}\left(l_{\Phi, p}\right)$ and every $k>1$, we have

$$
\frac{1}{2^{1 / p}} \leq d_{x_{1}, \ldots, x_{n}, k} \leq \frac{n k}{\min \left\{1,\left(k^{p}-1\right)^{1 / p}\right\}}=n k \max \left\{1, \frac{1}{\left(k^{p}-1\right)^{1 / p}}\right\}
$$

Indeed, if the first inequality does not hold then we could find $k>1$ and $\gamma>1$ such that $d_{x_{1}, \ldots, x_{n}, k}<\frac{1}{\gamma 2^{1 / p}}$. Then, by (3),

$$
\begin{aligned}
\sum_{j=1}^{n} I_{\Phi}\left(\frac{k x_{j}}{d_{x_{1}, \ldots, x_{n}, k}}\right) & \geq \sum_{j=1}^{n} I_{\Phi}\left(\frac{\gamma k x_{j}}{2^{-1 / p} \min \left\{\left\|x_{1}\right\|_{\Phi, p}, \ldots,\left\|x_{n}\right\|_{\Phi, p}\right\}}\right) \\
& \geq \gamma k \sum_{j=1}^{n} I_{\Phi}\left(\frac{x_{j}}{2^{-1 / p}\left\|x_{j}\right\|_{\Phi, p}}\right) \geq \gamma k \sum_{j=1}^{n} I_{\Phi}\left(\frac{x_{j}}{\left\|x_{j}\right\|_{\Phi}}\right) \\
& =\gamma k n \geq \gamma \max \left\{k,\left(k^{p}-1\right)^{1 / p}\right\} .
\end{aligned}
$$

Thus, for $1 \leq p<\infty$,

$$
\frac{1}{k} s_{p}\left(\sum_{j=1}^{n} I_{\Phi}\left(\frac{k x_{j}}{d_{x_{1}, \ldots, x_{n}, k}}\right)\right) \geq \frac{1}{k}\left(1+\gamma^{p} k^{p}\right)^{1 / p}>1
$$

and

$$
\frac{1}{k} s_{\infty}\left(\sum_{j=1}^{n} I_{\Phi}\left(\frac{k x_{j}}{d_{x_{1}, \ldots, x_{n}, k}}\right)\right) \geq \frac{1}{k} \max \{1, \gamma k\}=\gamma>1 .
$$

This contradicts the definition of the number $d_{x_{1}, \ldots, x_{n}, k}$ in (5). So, the first inequality in (6) holds.

Further, suppose that the second inequality does not hold. Then we could find $x_{1}, \ldots, x_{n} \in$ $S\left(l_{\Phi, p}\right), k>1$, and $\gamma>1$ such that $d_{x_{1}, \ldots, x_{n}, k}>\gamma \frac{n k}{\min \left\{1,\left(k^{p}-1\right)^{1 / p}\right\}}$. Since $\left\|x_{j}\right\|_{\Phi} \leq\left\|x_{j}\right\|_{\Phi, p}=1$, we have $I_{\Phi}\left(x_{j}\right) \leq\left\|x_{j}\right\|_{\Phi}=1$, whence

$$
\begin{aligned}
\sum_{j=1}^{n} I_{\Phi}\left(\frac{k x_{j}}{d_{x_{1}, \ldots, x_{n}, k}}\right) & \leq \sum_{j=1}^{n} I_{\Phi}\left(\frac{k \min \left\{1,\left(k^{p}-1\right)^{1 / p}\right\} x_{j}}{\gamma n k}\right) \\
& \leq \frac{\min \left\{1,\left(k^{p}-1\right)^{1 / p}\right\}}{\gamma n} \sum_{j=1}^{n} I_{\Phi}\left(x_{j}\right) \\
& \leq \frac{\min \left\{1,\left(k^{p}-1\right)^{1 / p}\right\}}{\gamma n} \sum_{j=1}^{n}\left\|x_{j}\right\|_{\Phi} \\
& \leq \frac{1}{\gamma} \min \left\{1,\left(k^{p}-1\right)^{1 / p}\right\} .
\end{aligned}
$$


Thus, for $1 \leq p<\infty$,

$$
\frac{1}{k} s_{p}\left(\sum_{j=1}^{n} I_{\Phi}\left(\frac{k x_{j}}{d_{x_{1}, \ldots, x_{n}, k}}\right)\right) \leq \frac{1}{k}\left(1+\frac{1}{\gamma^{p}}\left(k^{p}-1\right)\right)^{1 / p}<1
$$

and

$$
\frac{1}{k} s_{\infty}\left(\sum_{j=1}^{n} I_{\Phi}\left(\frac{k x_{j}}{d_{x_{1}, \ldots, x_{n}, k}}\right)\right) \leq \frac{1}{k} \max \left\{1, \frac{1}{\gamma}\right\}=\frac{1}{k}<1,
$$

and again we arrive at a contradiction with the definition of the number $d_{x_{1}, \ldots, x_{n}, k}$ in (5). Therefore the second inequality in (6) holds.

Now we will prove the main equality (4) of the theorem. For simplicity, denote

$$
d=\sup \left\{d_{x_{1}, \ldots, x_{n}}: x_{1}, \ldots, x_{n} \in S_{0}\left(l_{\Phi, p}\right) \text { and } x_{j} \wedge x_{j^{\prime}}=0 \text { for } j \neq j^{\prime}\right\}
$$

As an immediate consequence of (6), taking $k=2^{1 / p}$, we get $k^{p}-1=1$, so

$$
\frac{1}{2^{1 / p}}<d_{x_{1}, \ldots, x_{n}} \leq \inf _{k>1} \max \left\{n k, \frac{n k}{\left(k^{p}-1\right)^{1 / p}}\right\} \leq 2^{1 / p} n
$$

whence we infer that $0<2^{-1 / p}<d \leq 2^{1 / p} n<\infty$.

For any $0<\varepsilon<d$ we can find $x_{1}, \ldots, x_{n} \in S_{0}\left(l_{\Phi, p}\right)$, such that $x_{j} \wedge x_{j^{\prime}}=0$ for all $1 \leq j, j^{\prime} \leq n$, $j \neq j^{\prime}$ and $d_{x_{1}, \ldots, x_{n}, k}>d-\varepsilon$ for all $k>1$. Since the set $\left\{i \in \mathcal{N}: x_{j}(i) \neq 0\right\}, 1 \leq j \leq n$, is finite and the function $\Phi$ is continuous, we can find $k_{0}>0$ such that

$$
\left\|\bigvee_{j=1}^{n} \frac{x_{j}}{d-\varepsilon}\right\|_{\Phi, p}=\frac{1}{k_{0}} s_{p}\left(I_{\Phi}\left(k_{0} \bigvee_{j=1}^{n} \frac{x_{j}}{d-\varepsilon}\right)\right)
$$

If $k_{0} \leq 1$ then $\left\|\bigvee_{j=1}^{n} x_{j}\right\|_{\Phi, p} \geq d-\varepsilon$. On the other hand, if $k_{0}>1$ then we have

$$
\left\|\bigvee_{j=1}^{n} \frac{x_{j}}{d-\varepsilon}\right\|_{\Phi, p} \geq \frac{1}{k_{0}} s_{p}\left(\sum_{j=1}^{n} I_{\Phi}\left(\frac{k_{0} x_{j}}{d_{x_{1}, \ldots, x_{n}, k_{0}}}\right)\right)=1
$$

Thus $\left\|\bigvee_{j=1}^{n} x_{j}\right\|_{\Phi, p} \geq d-\varepsilon$ and, by the arbitrariness of $\varepsilon>0$, we get $\mu_{n}\left(l_{\Phi, p}\right) \geq d$.

Conversely, take any $x_{1}, \ldots, x_{n} \in S_{0}\left(l_{\Phi, p}\right)$ such that $x_{j} \wedge x_{j^{\prime}}=0$ for all $1 \leq j, j^{\prime} \leq n, j \neq j^{\prime}$ and let us take an arbitrary $\varepsilon>0$. Then $d_{x_{1}, \ldots, x_{n}}<d+\varepsilon$. Thus we can find $k_{1} \geq 1$ such that $d_{x_{1}, \ldots, x_{n}, k_{1}}<d+\varepsilon$ as well. By the definition of the $p$-Amemiya norm,

$$
\left\|\bigvee_{j=1}^{n} \frac{x_{j}}{d+\varepsilon}\right\|_{\Phi, p} \leq \frac{1}{k_{1}} s_{p}\left(I_{\Phi}\left(k_{1} \bigvee_{j=1}^{n} \frac{x_{j}}{d+\varepsilon}\right)\right) \leq \frac{1}{k_{1}} s_{p}\left(\sum_{j=1}^{n} I_{\Phi}\left(\frac{k_{1} x_{j}}{d_{x_{1}, \ldots, x_{n}, k_{1}}}\right)\right)=1
$$

Thus $\left\|\bigvee_{j=1}^{n} x_{j}\right\|_{\Phi, p} \leq d+\varepsilon$ and, by the arbitrariness of $\varepsilon>0$, we get $\mu_{n}\left(l_{\Phi, p}\right) \leq d$. Therefore, we have proved that $\mu_{n}\left(l_{\Phi, p}\right)=d$.

In order to prove important Theorem 2.3 we first need to prove the following result. 
Theorem 2.2 If $\Psi \notin \delta_{2}$ then, for any $1 \leq p \leq \infty$, the Orlicz space $l_{\Phi, p}$ contains an order almost isometric copy of $l_{1}$.

Proof Any linear functional $f \in\left(l_{\Phi, p}\right)^{*}$ can be uniquely expressed as $f=v+s$, where $v$ is belongs to the Köthe dual of $l_{\Phi}$ and $s$ is a singular functional on $l_{\Phi}$, that is, for any $x \in h_{\Phi}$ the equality $s(x)=0$ holds. By Lemma 1.49 in [19], $\|s\|=\sup \left\{s(x): I_{\Phi}(x)<\infty\right\}$. Further, by (2) and $\|v\|=\sup \left\{v(x):\|x\|_{\Phi, p} \leq 1\right\}$, we have

$$
\|v\|_{\Psi}=\sup \left\{v(x):\|x\|_{\Phi}^{0} \leq 1\right\} \leq\|v\| \leq\|v\|_{\Phi}^{0}=\sup \left\{v(x):\|x\|_{\Phi} \leq 1\right\} .
$$

Moreover, by Theorem 1.43 in [19] (see also [20]), $\lim _{n \rightarrow \infty}\left\|x-x_{n}\right\|_{\Phi}=\lim _{n \rightarrow \infty}\left\|x-x_{n}\right\|_{\Phi}^{0}=$ $\theta(v)$ for every $x \in \ell_{\Phi}$ and $x_{n}=\sum_{i=n}^{\infty} x(i) e_{i}$, where $\theta(v)=\inf \left\{\lambda>0: I_{\Phi}\left(\frac{v}{\lambda}\right)<\infty\right\}$. Hence, for any $v \in l_{\Psi}$, we have

$$
\lim _{n \rightarrow \infty}\left\|\sum_{i=n+1}^{\infty} v(i) e_{i}\right\|=\lim _{n \rightarrow \infty}\left\|\sum_{i=n+1}^{\infty} v(i) e_{i}\right\|_{\Psi}=\lim _{n \rightarrow \infty}\left\|\sum_{i=n+1}^{\infty} v(i) e_{i}\right\|_{\Psi}^{0}=\theta(v) .
$$

Since $\Psi \notin \delta_{2}$, there exists an element $v \in S\left(l_{\Psi}\right)$ such that $I_{\Psi}(\lambda x)=+\infty$ for every $\lambda \geq 1$, that is, $\theta(v)=1$. Therefore

$$
\lim _{n \rightarrow \infty}\left\|\sum_{i=n+1}^{\infty} v(i) e_{i}\right\|=1
$$

so, for any $\varepsilon>0$, there exists a sequence $i_{1}<i_{2}<\cdots$ such that $\left\|\sum_{i=i_{1}+1}^{\infty} v(i) e_{i}\right\|_{\Psi}^{0}<1+\varepsilon$ and, for all $j=1,2, \ldots$,

$$
1-\varepsilon<\left\|\sum_{i=i_{j}+1}^{i_{j+1}} v(i) e_{i}\right\|<1+\varepsilon
$$

Put $w_{j}=\sum_{i=i_{j}+1}^{i_{j+1}} v(i) e_{i}$ for $j \in \mathcal{N}$. Since $\left\|w_{j}\right\|=\sup \left\{w_{j}(x): x \in S\left(l_{\Phi, p}\right)\right\}$ and the number of elements of the support of $w_{j}$ is finite, for each $j \in \mathcal{N}$ there exists

$$
x_{j}=\sum_{i=i_{j}+1}^{i_{j+1}} x_{j}(i) e_{i} \in S\left(l_{\Phi, p}\right)
$$

such that $w_{j}\left(x_{j}\right)=\left\langle w_{j}, x_{j}\right\rangle=\sum_{i=i_{j}+1}^{i_{j+1}} x_{j}(i) v_{i}=\left\|w_{j}\right\|$ and $\left\langle w_{j}, x_{i}\right\rangle=0$ for every $j \neq i$. For any $a=(a(j)) \in l_{1}$, we have

$$
\left\|\sum_{j=1}^{\infty} a(j) x_{j}\right\|_{\Phi, p} \leq \sum_{j=1}^{\infty}|a(j)|\left\|x_{j}\right\|_{\Phi, p} \leq \sum_{j=1}^{\infty}|a(j)|=\|a\|_{l_{1}} .
$$

Since $\tilde{v}=\sum_{j=1}^{\infty} \operatorname{sign}(a(j)) w_{j}$ is a linear functional and

$$
\|\tilde{v}\|_{\Psi}^{0}=\left\|\sum_{j=1}^{\infty} \operatorname{sign}(a(j)) w_{j}\right\|_{\Psi}^{0}=\left\|\sum_{i=i_{1}}^{\infty} v(i) e_{i}\right\|_{\Psi}^{0} \leq 1+\varepsilon,
$$


we have $\tilde{v}(x) \leq(1+\varepsilon)\|x\|_{\Phi}$, so

$$
\begin{aligned}
(1+\varepsilon)\left\|\sum_{j=1}^{\infty} a(j) x_{j}\right\|_{\Phi, p} & \geq(1+\varepsilon)\left\|\sum_{j=1}^{\infty} a(j) x_{j}\right\|_{\Phi} \geq \tilde{v}\left(\sum_{i=1}^{\infty} a(i) x_{i}\right) \\
& =\sum_{j=1}^{\infty} \operatorname{sign}(a(j)) w_{j}\left(\sum_{i=1}^{\infty} a(i) x_{i}\right)=\sum_{j=1}^{\infty}|a(j)| w_{j}\left(x_{j}\right) \\
& =\sum_{j=1}^{\infty}|a(j)|\left\|w_{j}\right\| \geq(1-\varepsilon)\|a\|_{l_{1}} .
\end{aligned}
$$

By the arbitrariness of $\varepsilon>0$, we conclude that the Orlicz space $l_{\Phi, p}$ contains an order almost isometric copy of $l_{1}$.

Theorem 2.3 Assume that $\Phi(u) / u \rightarrow \infty$ as $u \rightarrow \infty$. For any $n \in \mathcal{N}$ and $1 \leq p \leq \infty$, $\mu_{n}\left(l_{\Phi, p}\right)=n$ if and only if $\Psi \notin \delta_{2}$.

Proof If $\Psi \notin \delta_{2}$ then, by Theorem 2.2, we immediately conclude that $\mu_{n}\left(l_{\Phi, p}\right)=\mu_{n}\left(l_{1}\right)=n$.

Now, let $\Psi \in \delta_{2}$. We will show that $\mu_{n}\left(l_{\Phi, p}\right)<n$. Let $x_{1}, \ldots, x_{n}$ be arbitrary elements of the set $S_{0}\left(l_{\Phi, p}\right)$ such that $x_{j} \wedge x_{j^{\prime}}=0$ for all $1 \leq j, j^{\prime} \leq n$ with $j \neq j^{\prime}$. Since the $\operatorname{set}\left\{i \in \mathcal{N}: x_{j}(i) \neq\right.$ $0,1 \leq j \leq n\}$ is finite and the function $\Phi$ is continuous, we can find a number $k \geq 1$, such that

$$
\frac{1}{k} s_{p}\left(\sum_{j=1}^{n} I_{\Phi}\left(k x_{j}\right)\right)=1
$$

Since $\Phi(u) / u \rightarrow \infty$ as $u \rightarrow \infty$, the function $\Psi$ takes only finite values. Since, moreover, $\Psi \in \delta_{2}$, for any $u_{0}>0$ we can find $\varepsilon>0$ such that $\Phi\left(\frac{u}{n-\varepsilon}\right) \leq \frac{1}{n} \Phi(u)$ for all $|u| \leq u_{0}$. Without loss of generality we can assume that $u_{0} \geq k \max \left\{\left|x_{j}(i)\right|: 1 \leq j \leq n, i \in \mathcal{N}\right\}$. We have $\frac{u}{n-\varepsilon} \leq$ $\Phi^{-1}\left(\frac{1}{n} \Phi(u)\right)$ for all $|u| \leq u_{0}$ (see $\left.[21,22]\right)$, so

$$
\sum_{j=1}^{n} I_{\Phi}\left(\frac{k x_{j}}{n-\varepsilon}\right) \leq \sum_{j=1}^{n} \sum_{i=n_{j}+1}^{n_{j+1}} \Phi\left(\Phi^{-1}\left(\frac{1}{n} \Phi\left(k x_{j}(i)\right)\right)\right)=\frac{1}{n} \sum_{j=1}^{n} I_{\Phi}\left(k x_{j}\right),
$$

whence

$$
\frac{1}{k} s_{p}\left(\sum_{j=1}^{n} I_{\Phi}\left(\frac{k x_{j}}{n-\varepsilon}\right)\right) \leq \frac{1}{k} s_{p}\left(\sum_{j=1}^{n} I_{\Phi}\left(k x_{j}\right)\right) \leq 1,
$$

so $d_{x_{1}, \ldots, x_{n}, k} \leq n-\varepsilon$. Thus, by the arbitrariness of the choice of elements $x_{1}, \ldots, x_{n} \in S_{\ell_{\Phi}, p}$ and by Theorem 2.1, $\mu_{n}\left(l_{\Phi, p}\right) \leq n-\varepsilon<n$.

\section{Fatou property, weak orthogonality, and weak fixed point property}

In order to generalize Maurey's (1980) proof of the w-FPP to a larger class of Banach lattices Borwein and Sims (1984) introduced the notion of a weakly orthogonal Banach lattice. A Banach lattice $X$ is said to be weakly orthogonal if for any sequence $\left(x_{n}\right)$ in $X_{+}$, 
which converges weakly to 0 we have

$$
\lim _{n \rightarrow \infty}\left\|\left|x_{n}\right| \wedge|x|\right\|=0
$$

for all $x \in X$. For the definition of Banach lattices we refer to [23]. Moreover, BetiukPilarska and Prus proved in [24] that weakly orthogonal Banach lattices, with the coefficient of monotonicity strictly smaller than 1 , have the weak fixed point property.

Sims (see [25]) introduced another definition of weak orthogonality of Banach lattices, denoted WORTH, and proved that if a Banach lattice is WORTH, then it satisfies non-strict Opial condition, that is, whenever $\left(x_{n}\right)$ is a weak-null sequence in $X$ then

$$
\liminf _{n \rightarrow \infty}\left\|x_{n}\right\| \leq \liminf _{n \rightarrow \infty}\left\|x_{n}+x\right\|
$$

for all $x \in X$. In [26] Cui, Hudzik and Płuciennik proved that Köthe sequence spaces with semi-Fatou property are WORTH if and only if they are order continuous. We will prove that, in the spaces $l_{\Phi, p}$, the criteria for the weak orthogonality due to Borwein and Sims are the same as for WORTH of Köthe spaces equipped with the semi-Fatou norms obtained in [26].

In the proof of Theorem 3.2 which gives the criteria for weak orthogonality of Orlicz sequence spaces, we will need the Fatou property of the space $l_{\Phi, p}$. Recall that a Banach lattice $X$ satisfies the Fatou property if and only if for every sequence $\left(x_{n}\right)$ with $0 \leq x_{n} \uparrow x$ we have $\left\|x_{n}\right\| \uparrow\|x\|$.

Theorem 3.1 For any $1 \leq p \leq \infty$ the Orlicz sequence space $l_{\Phi, p}$ satisfies the Fatou property.

Proof Let $\left(x_{n}\right)$ be a sequence of elements of $l_{\Phi, p}$ with $0 \leq x_{n} \uparrow x$. Evidently we can assume that $x \neq 0$. By the definition of the $p$-Amemiya norm, we can find a sequence $\left(k_{n}\right)$ of positive numbers such that

$$
\frac{1}{k_{n}}\left(1+I_{\Phi}^{p}\left(k_{n} x_{n}\right)\right)^{1 / p}-\frac{1}{n} \leq\left\|x_{n}\right\|_{\Phi, p} \leq \frac{1}{k_{n}}\left(1+I_{\Phi}^{p}\left(k_{n} x_{n}\right)\right)^{1 / p} .
$$

Suppose that the sequence $\left(k_{n}\right)$ is bounded. Without loss of generality we can assume that $k_{n} \rightarrow k_{0} \in[0, \infty)$. Since

$$
\frac{1}{k_{n}}-\frac{1}{n} \leq\left\|x_{n}\right\|_{\Phi, p} \leq\|x\|_{\Phi, p}<\infty
$$

we infer that $k_{0}>0$. Hence, by Fatou's lemma,

$$
\|x\|_{\Phi, p} \geq \lim _{n \rightarrow \infty}\left\|x_{n}\right\|_{\Phi, p} \geq \lim _{n \rightarrow \infty} \frac{1}{k_{n}}\left(1+I_{\Phi}^{p}\left(k_{n} x_{n}\right)\right)^{1 / p} \geq \frac{1}{k_{0}}\left(1+I_{\Phi}^{p}\left(k_{0} x\right)\right)^{1 / p} \geq\|x\|_{\Phi, p},
$$

i.e., $\left\|x_{n}\right\| \uparrow\|x\|$.

Now, assume that the sequence $\left(k_{n}\right)$ is not bounded. Without loss of generality we can assume that $k_{n} \uparrow \infty$ as $n \rightarrow \infty$. We claim that $\lim _{u \rightarrow \infty} \Phi(u) / u=c<\infty$. Indeed, if $\lim _{u \rightarrow \infty} \Phi(u) / u=\infty$ then

$$
\infty>\|x\|_{\Phi, p} \geq \lim _{n \rightarrow \infty}\left\|x_{n}\right\|_{\Phi, p} \geq \lim _{n \rightarrow \infty} \frac{I_{\Phi}\left(k_{n} x_{n}\right)}{k_{n}}=\lim _{n \rightarrow \infty} \sum_{\left\{i: x_{n}(i) \neq 0\right\}} \frac{\Phi\left(k_{n} x_{n}(i)\right)}{k_{n} x_{n}(i)} x_{n}(i),
$$


whence $x_{n}(i) \rightarrow 0$ as $n \rightarrow \infty$ for all $i \in \mathcal{N}$. Thus $x=0$ - a contradiction. Therefore, by subadditivity of the function $f(u)=u^{1 / p}$ on the interval $[0, \infty)$, we get

$$
\begin{aligned}
\|x\|_{\Phi, p} & \leq \lim _{k \rightarrow \infty} \frac{1}{k}\left(1+I_{\Phi}^{p}(k x)\right)^{1 / p} \leq \lim _{k \rightarrow \infty} \frac{1}{k}\left(1+I_{\Phi}(k x)\right)=\lim _{k \rightarrow \infty} \frac{1}{k} I_{\Phi}(k x) \\
& =\lim _{k \rightarrow \infty} \frac{1}{k} \sum_{\{i: x(i) \neq 0\}} \Phi(k x(i))=\lim _{k \rightarrow \infty} \sum_{\{i: x(i) \neq 0\}} \frac{\Phi(k x(i))}{k x(i)} x(i) \leq c\|x\|_{\ell^{1}} .
\end{aligned}
$$

Since $\frac{\Phi\left(k_{n} x_{n}(i)\right)}{k_{n} x_{n}(i)} x_{n}(i) \uparrow c x(i)$ as $n \rightarrow \infty$ for every $i \in \mathcal{N}$ with $x(i) \neq 0$, by the Beppo Levy theorem, we get

$$
\begin{aligned}
c\|x\|_{\ell^{1}} & \geq\|x\|_{\Phi, p} \geq \lim _{n \rightarrow \infty}\left\|x_{n}\right\|_{\Phi, p} \geq \lim _{n \rightarrow \infty} \frac{1}{k_{n}} I_{\Phi}\left(k_{n} x_{n}\right) \\
& \geq \lim _{n \rightarrow \infty} \sum_{\left\{i: x_{n}(i) \neq 0\right\}} \frac{\Phi\left(k_{n} x_{n}(i)\right)}{k_{n} x_{n}(i)} x_{n}(i)=\sum_{\{i: x(i) \neq 0\}} c x(i)=c\|x\|_{\ell^{1}},
\end{aligned}
$$

whence we conclude that, in the case of unbounded sequence $\left(k_{n}\right)$, the Fatou property holds as well.

Theorem 3.2 The Orlicz sequence space $l_{\Phi, p}(1 \leq p \leq \infty)$ is weakly orthogonal if and only if $\Phi \in \delta_{2}$.

Proof If $\Phi \notin \delta_{2}$ then there exists an element $x \in S_{+}\left(\ell_{\Phi, p}\right)$ satisfying $I_{\Phi}(\lambda x)=\infty$ for any $\lambda>1$. By the Fatou property we can find natural numbers $i_{1}<i_{2}<\cdots$ such that

$$
\frac{1}{2} \leq\left\|\sum_{i=i_{j}+1}^{i_{j+1}} x(i) e_{i}\right\|_{\Phi} \leq 1,
$$

and put $x_{j}=\sum_{i=i_{j+1}}^{i_{j+1}} x(i) e_{i}$. Then, by Lemma 2.3 in [27], we have $x_{j} \stackrel{w}{\rightarrow} 0$, but

$$
\lim _{j \rightarrow \infty}\left\|x_{j} \wedge x\right\|_{\Phi, p} \geq \lim _{j \rightarrow \infty}\left\|x_{j} \wedge x\right\|_{\Phi}=\lim _{j \rightarrow \infty}\left\|x_{j}\right\|_{\Phi} \geq \frac{1}{2}
$$

so the Orlicz space $l_{\Phi, p}$ is not weakly orthogonal - a contradiction.

Conversely, assume that $\Phi \in \delta_{2}$. Then the space $\ell_{\Phi}^{0}$ is order continuous, so for any $x \in l_{\Phi}^{0}$ and $\varepsilon>0$, there exists $i_{0} \in N$ such that $\left\|\sum_{i=i_{0}+1}^{\infty} x(i) e_{i}\right\|_{\Phi}^{0}<\varepsilon$. For any weakly null sequence $\left(x_{n}\right)$ in $l_{\Phi}^{0}$, there is $n_{0} \in N$ such that $\left\|\sum_{i=1}^{i_{0}} x_{n}(i) e_{i}\right\|_{\Phi}^{0}<\varepsilon$ whenever $n \geq n_{0}$, because weak convergence to zero implies its pointwise convergence to zero. Hence

$$
\left\|x_{n} \wedge x\right\|_{\Phi, p} \leq\left\|x_{n} \wedge x\right\|_{\Phi}^{0} \leq\left\|\sum_{i=1}^{i_{0}}\left|x_{n}(i)\right| e_{i}+\sum_{i=i_{0}+1}^{\infty}|x(i)| e_{i}\right\|_{\Phi}^{0} \leq 2 \varepsilon .
$$

By the arbitrariness of $\varepsilon$ we have $\lim _{n \rightarrow \infty}\left\|x_{n} \wedge x\right\|_{\Phi, p}=0$, so the Orlicz space $l_{\Phi, p}$ is weakly orthogonal.

Applying the result of Borwein and Sims from [7], which states that a weakly orthogonal Banach lattice with the Riesz angle less than 2 has the weak fixed point property, the fact 
that the space $\ell_{\Phi, p}$ is reflexive if and only if both functions $\Phi$ and $\Psi$ satisfy condition $\delta_{2}$ and Theorem 2.3, we get the following corollary.

Corollary 3.3 Every reflexive Orlicz sequence space $l_{\Phi, p}(1 \leq p \leq \infty)$ has the weak fixed point property, so they have the fixed point property as well.

\section{Dominguez-Benavides coefficient}

In 1994 Garcia-Falset [28] defined a geometric coefficient $R(X)$ which ensures the fixed point property and he obtained stability results for this coefficient. In particular, he proved that nearly uniformly smooth spaces have the FPP [29]. The coefficient $R(X)$ is defined as follows:

$$
R(X)=\sup \left\{\liminf _{n \rightarrow \infty}\left\|x_{n}+x\right\|\right\}
$$

where the supremum is taken over all $x \in B(X)$ and over all weakly null sequences of the unit ball $B(X)$.

In 1996 Dominguez-Benavides [30] generalized the coefficient $R(X)$ introducing,for all $a>0$, the condition

$$
R(a, X)=\sup \left\{\liminf _{n \rightarrow \infty}\left\|x_{n}+x\right\|\right\}
$$

where the supremum is taken over all $x \in X$ with $\|x\| \leq a$ and over all weakly null sequences of the unit ball $B(X)$ such that

$$
D\left(\left(x_{n}\right)\right)=\limsup _{n \rightarrow \infty}\left(\limsup _{m \rightarrow \infty}\left\|x_{n}-x_{m}\right\|\right) \leq 1
$$

The coefficients $R(a, X)$ play an important role in the fixed point theory for nonexpansive mappings. Moreover, $R(a, X)$ remains unaltered if we replace lim inf by lim sup in its definition. It was proved that, for a given $a \geq 0$, if $R(a, X)<1+a$ then the Banach space $X$ has the fixed point property. The following theorem provides a formula for $R(a, X)$ coefficients in the case of Orlicz sequence spaces $l_{\Phi, p}$.

Theorem 4.1 Assume that $\Phi(u) / u \rightarrow 0$ as $u \rightarrow 0$ and $\Phi$ satisfies the $\delta_{2}$ condition. For any $a>0$ we have

$$
R\left(a, l_{\Phi, p}\right)=\sup \left\{d_{x, z}: x \in G_{\Phi},\|z\|_{\Phi, p}=a\right\},
$$

where

$$
\begin{aligned}
& G_{\Phi}=\left\{x \in B\left(l_{\Phi, p}\right): \frac{1}{k} s_{p}\left(2 I_{\Phi}(k x)\right) \leq 1 \text { for some } k>1\right\}, \\
& d_{x, z}=\inf _{k>1}\left\{d_{x, z, k}>0: \frac{1}{k} s_{p}\left(I_{\Phi}\left(\frac{k x}{d_{x, z, k}}\right)+I_{\Phi}\left(\frac{k z}{d_{x, z, k}}\right)\right)=1\right\},
\end{aligned}
$$

and in each of the above defined sets only a finite number of coordinates of the sequences $x$, $z$ are non-zero. 
Let us note that $x \in G_{\Phi}$ whenever finite number of coordinates of $x$ are non-zero and $\|x\|_{\Phi, p} \leq \frac{1}{2}$. Indeed, in that case there exists $0<k_{0}<\infty$ such that $\frac{1}{k_{0}} s_{p}\left(I_{\Phi}\left(k_{0} x\right)\right)=\|x\|_{\Phi, p}$. If $k=k_{0} / 2$ then, by convexity of $\Phi$,

$$
\frac{1}{k} s_{p}\left(2 I_{\Phi}(k x)\right) \leq 2 \frac{1}{k_{0}} s_{p}\left(I_{\Phi}\left(k_{0} x\right)\right)=2\|x\|_{\Phi, p} \leq 1,
$$

i.e., $x \in G_{\Phi}$.

Note also that $R\left(a, l_{\Phi}\right) \geq a$. Indeed, taking $x=0$ for any $k>1$ and $z \in l_{\Phi}$ with $\|z\|_{\Phi, p}=a$, we have

$$
d_{0, z, k}=\frac{d_{0, z, k}}{k} s_{p}\left(I_{\Phi}\left(\frac{k z}{d_{0, z, k}}\right)\right) \geq\|z\|_{\Phi, p}=a .
$$

Thus $d_{0, z} \geq a$, whence $R\left(a, l_{\Phi}\right) \geq a$.

Proof Denote the right-hand side of the equality (9) by $d$. For any $0<\varepsilon<d$ there exist $x, z \in l_{\Phi, p}$ with finite supports, $x \in G_{\Phi},\|z\|_{\Phi, p}=a$, such that $d_{x, z}>d-\varepsilon$. Let $j \in \mathcal{N}$ be such a number that $x(i)=z(i)=0$ for every $i>j$. Define the sequence $\left(x_{n}\right)$ as follows:

$$
x_{n}=\sum_{i=n j+1}^{(n+1) j} x(i-n j) e_{i} .
$$

Since $\Phi(u) / u \rightarrow 0$ as $u \rightarrow 0$,

$$
\limsup _{\lambda \rightarrow 0} \frac{I_{\Phi}\left(\lambda x_{n}\right)}{\lambda}=\lim _{\lambda \rightarrow 0} \frac{I_{\Phi}(\lambda x)}{\lambda}=0 .
$$

Thus $\lim _{n \rightarrow \infty} x_{n}(i)=0$ for each $i \in \mathcal{N}$, whence we conclude that $x_{n}$ tends weakly to 0 (see $[19,27])$.

Since $x \in G_{\Phi}$, we can find $k_{0}>1$ such that $\frac{1}{k_{0}} s_{p}\left(2 I_{\Phi}\left(k_{0} x\right)\right) \leq 1$. Thus, for every $n, m \in \mathcal{N}$ we have

$$
\left\|x_{n}-x_{m}\right\|_{\Phi, p} \leq \frac{1}{k_{0}} s_{p}\left(I_{\Phi}\left(k_{0}\left(x_{n}-x_{m}\right)\right)\right) \leq \frac{1}{k_{0}} s_{p}\left(2 I_{\Phi}\left(k_{0} x\right)\right) \leq 1,
$$

whence $D\left(\left(x_{n}\right)\right) \leq 1$.

By the definition, $d_{x, z, k} \geq d_{x, z}>d-\varepsilon$ for every $k>1$. Since only a finite number of coordinates of the function $z$ are non-zero and $\lim _{n \rightarrow \infty} \inf \left\{i: x_{n}(i) \neq 0\right\} \rightarrow \infty$, we have $x_{n}(i) \cdot z(i)=0$ for all $i \in \mathcal{N}$ and all $n$ large enough, whence

$$
\begin{aligned}
1 & =\frac{1}{k} s_{p}\left(I_{\Phi}\left(\frac{k x}{d_{x, z, k}}\right)+I_{\Phi}\left(\frac{k z}{d_{x, z, k}}\right)\right)=\frac{1}{k} s_{p}\left(I_{\Phi}\left(\frac{k x_{n}}{d_{x, z, k}}\right)+I_{\Phi}\left(\frac{k z}{d_{x, z, k}}\right)\right) \\
& \leq \frac{1}{k} s_{p}\left(I_{\Phi}\left(\frac{k x_{n}}{d-\varepsilon}\right)+I_{\Phi}\left(\frac{k z}{d-\varepsilon}\right)\right)=\frac{1}{k} s_{p}\left(I_{\Phi}\left(\frac{k\left(x_{n}+z\right)}{d-\varepsilon}\right)\right)
\end{aligned}
$$

for all $n$ large enough. Hence

$$
\left\|\frac{x_{n}+z}{d-\varepsilon}\right\|_{\Phi, p}=\inf _{k>1} \frac{1}{k} s_{p}\left(I_{\Phi}\left(\frac{k\left(x_{n}+z\right)}{d-\varepsilon}\right)\right) \geq 1,
$$


i.e.,

$$
\liminf _{n \rightarrow \infty}\left\|x_{n}+z\right\|_{\Phi, p} \geq d-\varepsilon
$$

By the arbitrariness of $\varepsilon>0$ we get $R\left(a, l_{\Phi}\right) \geq d$.

Now we will prove that $R\left(a, l_{\Phi}\right) \leq d$. Assume that $\left(x_{n}\right)$ is a weakly null sequence of elements of $B\left(l_{\Phi, p}\right)$ with $D\left(\left(x_{n}\right)\right) \leq 1$. Let $\varepsilon>0$ and put $n_{1}=1$. Since $\Phi \in \delta_{2}(0)$, the norm $\|\cdot\|_{\Phi, p}$ is order continuous in $l_{\Phi, p}$, so we can find $i_{1} \in \mathcal{N}$ such that

$$
\left\|\sum_{i=i_{1}+1}^{\infty} x_{n_{1}}(i) e_{i}\right\|_{\Phi, p}<\varepsilon
$$

Since, for every $i \in \mathcal{N}, \lim _{n \rightarrow \infty} x_{n}(i)=0$, there exists $n_{2}>n_{1}$ such that

$$
\left\|\sum_{i=1}^{i_{1}} x_{n_{2}}(i) e_{i}\right\|_{\Phi, p}<\varepsilon
$$

Since $x_{n_{2}}$ has an absolutely continuous norm, there exists $i_{2}>i_{1}$ such that

$$
\left\|\sum_{i=i_{2}+1}^{\infty} x_{n_{2}}(i) e_{i}\right\|_{\Phi, p}<\varepsilon .
$$

In this way we can find, by induction, two sequences $\left(i_{k}\right)$ and $\left(n_{j}\right)$ such that

$$
\left\|\sum_{i=i_{j}+1}^{\infty} x_{n_{j}}(i) e_{i}\right\|_{\Phi, p}<\varepsilon \text { and }\left\|\sum_{i=1}^{i_{j}} x_{n_{j+1}}(i) e_{i}\right\|_{\Phi, p}<\varepsilon .
$$

For any $j \in \mathcal{N}$ put $y_{j}=\sum_{i=i_{j-1}+1}^{i_{j}} x_{n_{j}}(i) e_{i}$. Since $y_{k}(i) \cdot y_{s}(i)=0$ for every $i, k, s \in N, k \neq s$, we have

$$
\begin{aligned}
\left\|y_{k}-y_{s}\right\|_{\Phi, p} \leq & \left\|x_{n_{k}}-x_{n_{s}}\right\|_{\Phi, p}+\left\|\sum_{i=1}^{i_{k-1}} x_{n_{k}}(i) e_{i}\right\|_{\Phi, p}+\left\|\sum_{i=1}^{i_{s-1}} x_{n_{s}}(i) e_{i}\right\|_{\Phi, p} \\
+ & \left\|\sum_{i=i_{k}+1}^{\infty} x_{n_{k}}(i) e_{i}\right\|_{\Phi, p}+\left\|\sum_{i=i_{s}+1}^{\infty} x_{n_{s}}(i) e_{i}\right\|_{\Phi, p} \leq D\left(\left(x_{n}\right)\right)+4 \varepsilon \leq 1+4 \varepsilon .
\end{aligned}
$$

Let $z_{j}=\frac{y_{j}}{1+4 \varepsilon}$ for $j \in \mathcal{N}$. Then, by the last inequalities, $D\left(\left(z_{j}\right)\right) \leq 1$ and $\lim _{j \rightarrow \infty} z_{j}(i)=0$. Thus

$$
\lim _{\lambda \rightarrow 0} \sup _{j} \frac{I_{\Phi}\left(\lambda z_{j}\right)}{\lambda} \leq \lim _{\lambda \rightarrow 0} \sup _{j} \frac{I_{\Phi}\left(\lambda x_{n_{j}}\right)}{\lambda}=0
$$

whence the sequence $\left(z_{j}\right)$ converges weakly to 0 (see $\left.[19,27]\right)$.

Since $D\left(\left(z_{j}\right)\right) \leq 1$, there exists $l_{0}>1$ such that

$$
\frac{1}{l_{0}} s_{p}\left(I_{\Phi}\left(l_{0} z_{k}\right)+I_{\Phi}\left(l_{0} z_{s}\right)\right) \leq 1,
$$


i.e.,

$$
\begin{aligned}
& I_{\Phi}\left(l_{0} z_{k}\right)+I_{\Phi}\left(l_{0} z_{s}\right) \leq\left(l_{0}^{p}-1\right)^{1 / p} \text { for } 1 \leq p<\infty, \\
& I_{\Phi}\left(l_{0} z_{k}\right)+I_{\Phi}\left(l_{0} z_{s}\right) \leq l_{0} \text { for } p=\infty
\end{aligned}
$$

for every $k, s \in N, k \neq s$. Then, for $1 \leq p<\infty$, either $I_{\Phi}\left(l_{0} z_{k}\right) \leq \frac{1}{2}\left(l_{0}^{p}-1\right)^{1 / p}$ or $I_{\Phi}\left(l_{0} z_{s}\right) \leq$ $\frac{1}{2}\left(l_{0}^{p}-1\right)^{1 / p}$ and, for $p=\infty$, either $I_{\Phi}\left(l_{0} z_{k}\right) \leq \frac{1}{2} l_{0}$ or $I_{\Phi}\left(l_{0} z_{s}\right) \leq \frac{1}{2} l_{0}$. Hence, passing to a subsequence if necessary, we can assume that $z_{k} \in G_{\Phi}$ for every $k \in \mathcal{N}$.

Now take any $x \in l_{\Phi}$ with finite support such that $\|x\|_{\Phi, p}=a$. Then $x(i) \cdot z_{k}(j)=0$ for every $i, j \in \mathcal{N}$ and every $k \in N$ large enough. Thus, for every $h>1$ we have

$$
1=\frac{1}{h} s_{p}\left(I_{\Phi}\left(\frac{h z_{k}}{d_{x, z_{k}, h}}\right)+I_{\Phi}\left(\frac{h x}{d_{x, z_{k}, h}}\right)\right)=\frac{1}{h} s_{p}\left(I_{\Phi}\left(\frac{h\left(x+z_{k}\right)}{d_{x, z_{k}, h}}\right)\right),
$$

whence $\left\|x+z_{k}\right\|_{\Phi, p} \leq \inf _{h>1} d_{x, z_{k}, h}=d_{x, z_{k}}$ for every $k \in \mathcal{N}$ large enough. By the arbitrariness of suitable $x$ and $\left(z_{k}\right)$ we conclude that $R\left(a, l_{\Phi, p}\right) \leq d$.

\section{Competing interests}

The authors declare that they have no competing financial interests.

\section{Authors' contributions}

All authors made substantial contributions to all of the following: (1) the conception and design of the study, (2) drafting the article or revising it critically for important intellectual content, (3) final approval of the version to be submitted.

\section{Author details}

'Department of Mathematics, Harbin University of Science and Technology, Xuefu Road 52, Harbin, 150080, P.R. China ${ }^{2}$ Faculty of Mathematics and Computer Science, Adam Mickiewicz University in Poznań, ul. Umultowska 87, Poznań, 61-614, Poland.

\section{Acknowledgements}

All the authors were supported by the National Science Centre, Poland, Grant no. UMO-2012/07/B/ST1/03360.

Received: 7 May 2016 Accepted: 11 August 2016 Published online: 06 September 2016

\section{References}

1. Goebel, K, Kirk, W: Topics in Metric Fixed Point Theory. Cambridge University Press, Cambridge (1991)

2. Kirk, WA, Sims, B (eds.): Handbook of Metric Fixed Point Theory. Kluwer Academic, Dordrecht (2001)

3. Abramovich, YA, Lozanovskiǐ, GY: Certain numerical characteristics of KN-lineals. Mat. Zametki 14(5), 723-732 (1973) (in Russian)

4. Lifshits, EA: On the theory of partially ordered Banach spaces. Funkc. Anal. Ego Prilož. 3(1), $91-92$ (1969) (in Russian)

5. Teskrekas, PC: Some applications of L-constants and M-constants on Banach lattices. J. Lond. Math. Soc. 18(2), 133-139 (1978)

6. Wnuk, W: Remarks concerning M-constants in Banach lattices. In: Kato, M, Maligranda, L, Suzuki, T (eds.) Proc. of the Fourth International Symposium on Banach and Function Spaces, Kitakyusiu, Japan, pp. 259-273. Yokohama Publishers, Yokohama (2014)

7. Borwein, J, Sims, B: Non-expansive mappings on Banach lattices and related topics. Houst. J. Math. 10(3), 339-356 (1984)

8. Luxemburg, WAJ: Banach function spaces. Thesis, Delft (1955)

9. Musielak, J: Orlicz Spaces and Modular Spaces. Lecture Notes in Math., vol. 1034. Springer, Berlin (1983)

10. Rao, RR, Ren, ZD: Theory of Orlicz Spaces. Dekker, New York (1991)

11. Orlicz, W: Über eine gewisse Klasse von Räumen von Typus B. Bull. Acad. Pol. A 8/9, 207-220 (1932)

12. Maligranda, L: Orlicz Spaces and Interpolation. Seminars in Mathematics, vol. 5. Universidade Estadual de Campinas, São Paulo (1989)

13. Hudzik, H, Maligranda, L: Amemyia norm equals Orlicz norm in general. Indag. Math. 11(4), 573-585 (2000)

14. Cui, YA, Duan, L, Hudzik, H, Wisła, M: Basic theory of $p$-Amemiya norm in Orlicz spaces $(1 \leq p \leq \infty)$ : extreme points and rotundity in Orlicz spaces equipped with these norms. Nonlinear Anal. 69, 1796-1816 (2008)

15. Cui, YA, Hudzik, H, Li, J, Wisła, M: Strongly extreme points in Orlicz spaces equipped with the $p$-Amemiya norm. Nonlinear Anal., Theory Methods Appl. 71, 6343-6364 (2009)

16. Cui, YA, Hudzik, H, Wisła, M: Monotonicity properties and dominated best approximation problems in Orlicz spaces equipped with the $p$-Amemiya norm. J. Math. Anal. Appl. 432, 1095-1105 (2015)

17. Cui, YA, Hudzik, H, Wisła, M, Wlaźlak, K: Non-squareness properties of Orlicz spaces equipped with the $p$-Amemiya norm. Nonlinear Anal., Theory Methods Appl. 75, 3973-3993 (2012) 
18. Wisła, M: Geometric properties of Orlicz spaces equipped with p-Amemiya norms - results and open questions. Comment. Math. 55(2), 183-209 (2015)

19. Chen, ST: Geometry of Orlicz spaces. Diss. Math. 356, 1-204 (1996)

20. Hudzik, H, Zbąszyniak, Z: Smoothness in Musielak-Orlicz spaces equipped with the Orlicz norm. Collect. Math. 48(4-6), 543-561 (1997)

21. Chen, ST, Hudzik, H: On some convexities of Orlicz and Orlicz-Bochner spaces. Comment. Math. Univ. Carol. 29(1), 13-29 (1988)

22. Hudzik, H: Uniformly non- $\ell_{n}^{(1)}$ Orlicz spaces with Luxemburg norm. Stud. Math. 81(3), 271-284 (1985)

23. Lindenstrauss, J, Tzafriri, L: Classical Banach Spaces. I. Sequence Spaces. Springer, Berlin (1977)

24. Betiuk-Pilarska, A, Prus, S: Banach lattices which are order uniformly noncreasy. J. Math. Anal. Appl. 342, 1271-1279 (2008)

25. Sims, B: Orthogonality and fixed points of nonexpansive maps. Proc. Cent. Anal. Aust. Nat. Univ. 20, 178-186 (1988)

26. Cui, YA, Hudzik, H, Płuciennik, R: Weak orthogonality and weak property $(\beta)$ in some Banach sequence spaces. Czechoslov. Math. J. 49(124), 303-316 (1999)

27. Cui, YA, Hudzik, H, Wisła, M, Zhao, L: Some geometric coefficients in Musielak-Orlicz sequence spaces equipped with the Luxemburg norm. In: Proceedings of the Seventh International Conference on Fixed Point Theory and Its Applications, Guanajuato, Mexico, July 17-23, pp. 49-62 (2005)

28. Garcia-Falset, J: Stability and fixed points for nonexpansive mappings. Houst. J. Math. 10, 495-506 (1994)

29. Garcia-Falset, J: The fixed point in Banach spaces with NUS property. J. Math. Anal. Appl. 215, 532-542 (1997)

30. Dominguez-Benavides, T: A geometrical coefficient implying the fixed point property and stability results. Houst. J. Math. 22, $835-849$ (1996)

\section{Submit your manuscript to a SpringerOpen ${ }^{\circ}$ journal and benefit from:}

- Convenient online submission

Rigorous peer review

- Immediate publication on acceptance

- Open access: articles freely available online

- High visibility within the field

- Retaining the copyright to your article 